\title{
One-flow syntheses of diverse heterocyclic furan chemicals directly from fructose via tandem transformation platform
}

\author{
Guan-Young Jeong ${ }^{1}$, Ajay K Singh ${ }^{1}$, Siddharth Sharma ${ }^{2}$, Ki Won Gyak ${ }^{1}$, Ram Awatar Maurya ${ }^{3}$ and \\ Dong-Pyo Kim ${ }^{1}$
}

The sustainable green chemistry associated with lignocellulosic biomass is of current interest for producing various chemical feedstocks via multi-step transformation processes. Here we introduce a chemical platform system for the multicomponent cascade transformation of natural lignocellulosic biomass resources. We demonstrate the concept by developing an integrated continuous two-step microfluidic system as a tandem transformation platform for direct conversion of fructose to diverse furan chemicals with excellent yields up to $99 \%$ via decarbonylation, etherification, oxidation and hydrogenolysis of a 5-hydroxymethylfurfural (HMF) intermediate. A sequential two-step process is utilized to complete the dehydration of fructose in the surface acid catalyst at $150^{\circ} \mathrm{C}$ for $6 \mathrm{~min}$, which is followed by the four types of HMF conversion in a binary or ternary phase to produce furfuryl alcohol (94\% yield), 5-ethoxymethylfurfural (99\%), 2,5-diformylfuran (82\%) and 2,5-dimethylfuran (90\%) with magnetic-based heterogeneous catalysts at $70-150^{\circ} \mathrm{C}$ for $6-60 \mathrm{~min}$. This innovative tandem microfluidic platform enables precise control of the reaction temperature and time for each individual biomass conversion step in a one-flow manner with no separation and purification steps for intermediates and catalysts.

NPG Asia Materials (2015) 7, e173; doi:10.1038/am.2015.21; published online 10 April 2015

\section{INTRODUCTION}

The efficient transformation of abundant biomass resources such as carbohydrates, lignin, fatty acids, lipids and cellulose into organic chemicals has received much attention as a path to green chemistry that is environmentally friendly. ${ }^{1}$ Among six-carboned carbohydrates, hexoses are the most abundant monosaccharides existing in nature. ${ }^{2,3}$ Of these, fructose and glucose are suitable for the use as an economical chemical feedstock for producing heterocyclic furan chemicals such as 5-hydroxymethylfurfural (5-HMF), ${ }^{4}$ furfuryl alcohol (FFA), ${ }^{5}$ 2,5-diformylfuran (2,5-DFF $)^{6}$ and 2,5-dimethylfuran (2,5-DMF). ${ }^{3,7}$ It is known that the catalytic transformation of hexoses into these furanic products involves several chemical steps such as dehydration, hydrolysis, isomerization, condensation, hydrogenation and oxidation. ${ }^{4}$ In general, 5-HMF, a dehydration product of fructose, is an important renewable chemical intermediate that can be used as a starting material for producing diverse furan building blocks, which can be used as fuel or replacements of oil-derived chemicals. ${ }^{4}$ The synthesis of furan derivatives from carbohydrates typically involves multiple reaction steps, such as dehydration for HMF production, followed by further transformation in the presence of heterogeneous catalysts. ${ }^{4,8}$ The synthesis of furan derivatives from HMF in a single step is well documented. However, the direct synthesis of the derivatives from carbohydrates is rarely reported and remains challenging because it requires a two-step process involving the isolation and purification of HMF as an intermediate and separation of the catalyst from the main stream, which is a time-, cost- and labor-consuming process. ${ }^{6}$ A onepot catalytic process has been developed to remove the intermediate purification step in the multi-step syntheses. ${ }^{6,8,9}$ However, the one-pot reaction generally requires a long reaction time extending to several tens of hours that often causes thermal degradation of the highly reactive furan ring molecules. ${ }^{6,8} \mathrm{~A}$ set of catalysts in one pot could result in negative interference between catalysts, resulting in low efficiency of productivity. ${ }^{10}$ Although the multi-functional catalysts allow several reaction steps to be completed in one reactor and avoid a costly intermediate separation process, ${ }^{11}$ the recycling of the catalyst still remains an active topic in the downstream chemical processing technology. Recently, we reported the concept and method for continuous and integrated in situ generation on the demand, separation and reaction of malodor, toxic and/or explosive reagents utilizing microfluidic systems. ${ }^{12-14}$ These approaches illustrated the advantages of forming and immediately utilizing the intermediates for subsequent reactions in a continuous-flow system. The successful outcome suggests that an integrated continuous-flow system is suitable for direct conversion of carbohydrate resources into organic chemicals in

${ }^{1}$ Department of Chemical Engineering, National Center of Applied Microfluidic Chemistry, POSTECH (Pohang University of Science \& Technology), Pohang, Korea; ${ }^{2}$ Department of Chemistry, Guru Nanak Dev University, Amritsar, India and ${ }^{3}$ Division of Natural Product Chemistry, CSIR-Indian Institute of Chemical Technology, Hyderabad, India Correspondence: Professor D-P Kim, Department of Chemical Engineering, National Center of Applied Microfluidic Chemistry, POSTECH (Pohang University of Science and Technology), Environmental Engineering Building, San 31, Hyoja-dong, Pohang 790-784, Korea.

E-mail: dpkim@postech.ac.kr

Received 11 January 2015; revised 5 February 2015; accepted 9 February 2015 


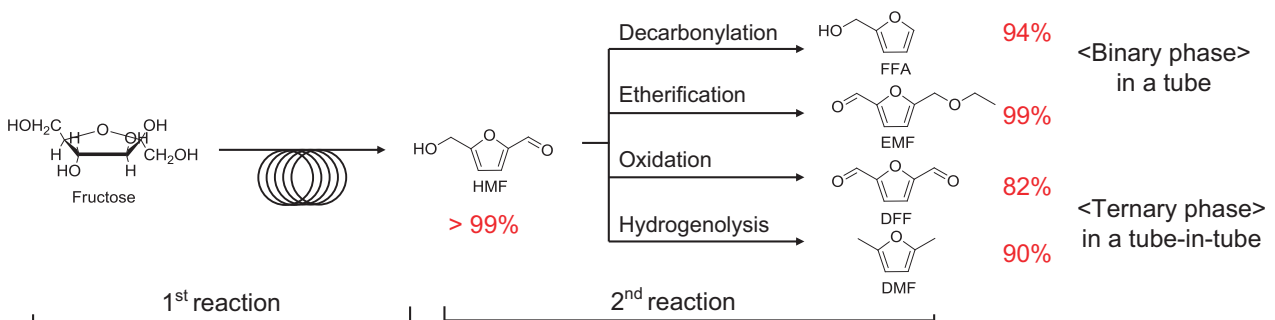

Integrated Microfluidic Transformation Platform

Scheme 1 Step-wise conversion of D-fructose carbohydrates into the four types of furanics via 5-hydroxymethylfurfural (HMF).

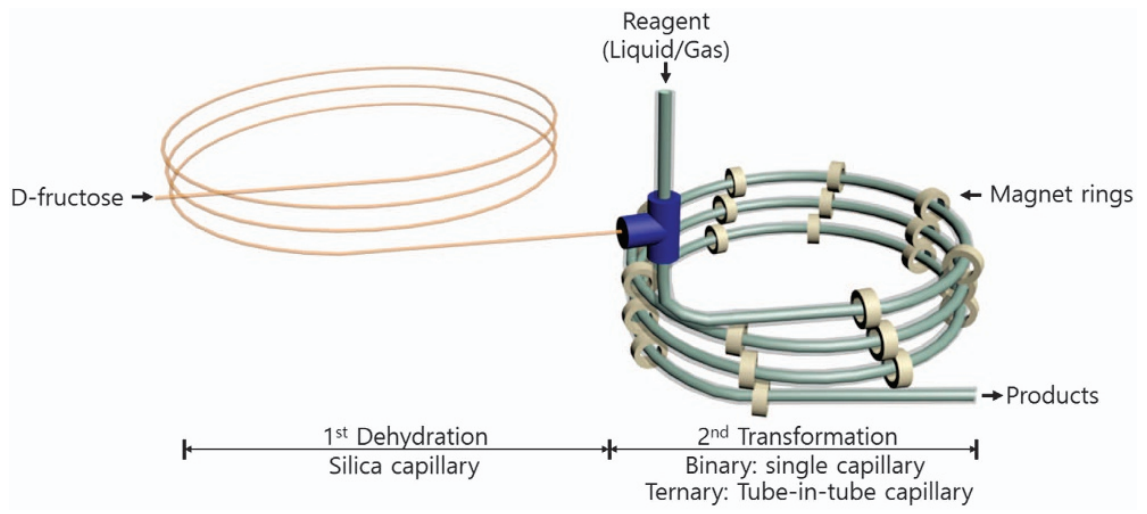

Figure 1 Illustrative scheme of integrated multi-step microreactor system for direct conversion of fructose into furanics.

an efficient manner. The intrinsic advantages of the microfluidic system, such as enhanced heat transfer due to the high surface to volume ratio and efficient mixing due to the short diffusion paths, facilitate control of the C6 carbohydrates with low thermal stability. ${ }^{15-20}$ In particular, the facile control of the retention time by varying the flow rate may prevent over-reactions from producing the unwanted black char residue. In addition, it is expected that the heterogeneous catalysts are readily immobilized in the microchannel and recycled without the loss of high stability, which eliminates the catalyst separation step. Moreover, the integrated microfluidic system could be constructed for various continuous-flow and multi-step chemical processes by simply assembling different catalyst immobilized microfluidic units using T-junctions. Hence, one-pot chemistry in biomass conversion can be synergistically realized by a consecutive series of biomass-based organic reactions without the use of sophisticated tandem catalysts.

In this work, we describe a continuous-flow and integrated microfluidic transformation platform for efficient and direct conversion of fructose biomass into furan derivatives without separation of HMF and catalysts. A set of heterogeneous catalysts for multi-step synthetic processes is serially immobilized at the inner wall surface of the microreactor by surface modification or magnetization, which eliminates catalyst separation as well as chemical interference in the downstream chemical operation. Indeed, excellent conversion of fructose to FFA in $94 \%$ yield, 5-ethoxymethylfurfural (EMF) in 99\%, DFF in $82 \%$ yield and DMF in $90 \%$ yield, respectively, were achieved by the sequential two-step process via in situ utilization of HMF, as summarized in Scheme 1. This tandem strategy integrated the first step of complete dehydration of fructose to HMF by surface sulfonic acid catalyst at $150{ }^{\circ} \mathrm{C}$ for $6 \mathrm{~min}$ with the subsequent step of binary phase reactions of decarbonylation and etherification, ternary liquid-gas-solid phase reactions of oxidation and hydrogenolysis of HMF in the tube-in- tube system to respective furan compounds, which was performed in the presence of three mixed-phase catalysts $\left(\mathrm{Pd} / \mathrm{Fe}_{3} \mathrm{O}_{4} / \mathrm{SiO}_{2}, \mathrm{Fe}_{3} \mathrm{O}_{4} / \mathrm{rGO}\right.$ and $\mathrm{Ru} / \mathrm{Cu} / \mathrm{Fe}_{3} \mathrm{O}_{4} / \mathrm{N}$-doped graphene oxide $(\mathrm{N}-\mathrm{rGO})$ ) and one coreshell-type catalyst $\left(\mathrm{Fe}_{3} \mathrm{O}_{4} @ \mathrm{SiO}_{2} @ \mathrm{Mn}\right)$ at different temperatures (150 and $70^{\circ} \mathrm{C}$ ) and retention times $(12-70 \mathrm{~min})$.

In particular, the supported catalysts on the magnetic $\mathrm{Fe}_{3} \mathrm{O}_{4}$ particles could easily be positioned in appropriate amounts at the inner wall of the microchannel by an external magnet for specific chemical reactions and control. This integrated multi-step microfluidic system successfully demonstrated direct biomass conversion with high selectivity in a time-, cost- and labor-saving manner with no catalyst recycle and without the tedious separation-extraction step of intermediates.

\section{MATERIALS AND METHODS}

Chemical reagents including dimethylsulfoxide, hydrogen peroxide, sulfuric acid, deionized water, ethanol, ammonia solution and hydrochloric acid were purchased from Daejung Chemicals (Siheung, Korea); D-fructose, 5-HMF (98\%), FFA (99\%), 3-mercaptopropyltrimethoxysilane (3-MPTMS), EMF, 2,5DMF, ruthenium chloride $\left(\mathrm{RuCl}_{2}\right)$, copper chloride $(\mathrm{CuCl})$, aminopropyltriethoxysilane (APTES), tetraethoxyorthosilane (TEOS), manganese acetate $\left(\mathrm{Mn}(\mathrm{OAC})_{3}\right)$, iron(II) chloride $\left(\mathrm{FeCl}_{2}\right)$, iron(III) chloride $\left(\mathrm{FeCl}_{3}\right)$, salicylaldehyde, zinc chloride $\left(\mathrm{ZnCl}_{2}\right)$, formaldehyde (38\%), graphite powder, potassium permanganate, hydrazine hydrate and poly(ethylene glycol)-block-poly(propylene glycol)-block-poly(ethylene glycol) (P123 (EO20PO70EO20) tri-block copolymer) were purchased from Sigma Aldrich (St Louis, MO, USA); and vanadium sulfate $\left(\mathrm{VOSO}_{4}\right)$ was purchased from Alfa Aesar (Ward Hill, MA, USA).

\section{RESULTS AND DISCUSSION}

Integrated microfluidic transformation platform system

The illustration of an integrated multi-step microfluidic system is shown in Figure 1. The integrated continuous synthesis system 
consists of the two units of capillary microreactors. For the first step of dehydration, the conversion of D-fructose to HMF, sulfonic acid as a dehydration catalyst is immobilized on the fused silica capillary wall by functionalizing the hydroxyl groups on the capillary. ${ }^{21}$ The inner surface of the silica-fused capillary $(150-\mu \mathrm{m}$ id, $200-\mathrm{cm}$ length) was modified by 3-MPTMS, and then, the resulting - $\mathrm{SH}$ groups were oxidized by hydrogen peroxide to form sulfonic acid, as reported in the literature (see ESI Supplementary Figure S3 and the experimental part for details). ${ }^{22}$ Dimethylsulfoxide was selected as the solvent because it is compatible with both the catalytic dehydration and subsequent downstream steps. ${ }^{3}$ A solution of D-fructose in dimethylsulfoxide $(0.5 \mathrm{M})$ was injected into the modified silica capillary using a high-pressure pump to control the flow driving force. The reaction temperature and residence time were controlled by immersing the silica capillary in the silicone oil bath on a heater and by varying the flow rate or the loop length of the capillary tube.

Table 1 shows the results on the catalytic dehydration of D-fructose to HMF in the sulfonic acid-functionalized silica capillary under various conditions. The conversion at $120{ }^{\circ} \mathrm{C}$ increased from $63 \%$ (yield of $57 \%$ ) to $84 \%$ (yield of $80 \%$ ) as the residence time was varied from 3 to $30 \mathrm{~min}$, respectively, by manipulating the flow rate. Upon increasing the reaction temperature to $150{ }^{\circ} \mathrm{C}$, both the conversion and yield of HMF reached up to $99 \%$ in only 6 min of retention time, which is much more efficient than the reported conventional batch process $(100 \%$ conversion with $76 \%$ yield for $2 \mathrm{~h}){ }^{3}$ The complete conversion in a relatively short reaction time is attributed to efficient mixing and isothermal conditions with no hot spots due to the excellent mass and heat transfer that originate from the high surface area to volume ratio, as is well documented. ${ }^{16}$ The acid-catalyst amount was determined by measuring the difference in the 3-MPTMS concentration using ICP-MS before/after the introduction of flow. The result indicated that $2 \mathrm{mmol}$ sulfonic acid was immobilized in the silica capillary reactor when $500 \mathrm{mmol} 3$-MPTMS solution was introduced into the silica-fused capillary at $20 \mu \mathrm{min}^{-1}$ at room temperature (collected $498 \mathrm{mmol} 3$-MPTMS at the outlet).

The second capillary reactor produces furan compounds by in situ transformation of the HMF produced in the first reactor unit. Magnetic-based solid catalysts for the conversion are immobilized in an appropriate amount on the inner wall by external magnet rings (NdFeB type, 25-50 MGOe). The on-demand magnetic-controlled catalyst loading is a certain advantage for varying the amount with the number of magnetic rings and the controllable location for different classes of organic reactions and also eliminating the need to separate

Table 1 Catalytic dehydration of D-fructose into HMF in sulfonic acidfunctionalized silica capillary microreactor ( $0.5 \mathrm{M}$ reagent in DMSO)

\begin{tabular}{cccccc}
\hline Entry & Temp $\left({ }^{\circ} \mathrm{C}\right)$ & Res. time $(\mathrm{min})$ & Flow rate $\left(\mu / \mathrm{min}^{-1}\right)$ & Conv. (\%) & Yield (\%) \\
\hline 1 & 120 & 30 & 1 & $84(61)$ & $80(20)$ \\
2 & 120 & 10 & 3 & 80 & 74 \\
3 & 120 & 6 & 5 & 72 & 66 \\
4 & 120 & 3 & 10 & 63 & 57 \\
5 & 150 & 30 & 1 & $99(68)$ & $99(27)$ \\
6 & 150 & 10 & 3 & 99 & 99 \\
7 & 150 & 6 & 5 & 99 & 99 \\
8 & 150 & 3 & 10 & 92 & 91
\end{tabular}

Abbreviations: Conv., conversion; DMSO, dimethylsulfoxide; HMF, 5-hydroxymethylfurfural; Res. time, residence time; Temp, temperature.

The conversion and yield in parentheses (entries 1 and 5) were those in the absence of the functionalized sulfonic acid catalyst. All the entries of conversion and yield were characterized functionalized sulfonic acid catalyst. All the en
by GC-MS using THF as an internal standard. the catalyst for recycling or to purify the products. This automated continuous-flow protocol allows time-, cost- and labor-saving synthesis of valuable organic compounds from inexpensive fructose with no isolation step of the intermediate product of HMF. In the actual process, each step was individually tested to optimize the chemical performance for the most suitable reaction temperatures and times and then integrated to conduct the two-step process in a continuous-flow manner.

\section{Binary phase conversion to furan chemicals from fructose}

The complete conversion and yield of HMF proved the usefulness of the first silica capillary microreactor. The second reactor connected to the first in this integrated platform system transforms HMF. Catalytic conversion of HMF to FFA was selected as the first example to test the efficiency of the integrated two-step microfluidic system. ${ }^{23}$ This alcohol has been used in solvents, fragrance chemicals and rocket fuel and in the production of resins and pharmaceutics. To date, various heterogeneous catalytic conversions of HMF to FFA in bulk synthesis at $130-220^{\circ} \mathrm{C}$ have suffered from a few problems such as thermal degradation of HMF because of the prolonged reaction at high temperature and the need for a large amount of active metal catalyst. $^{5,23}$

For the integrated continuous-flow synthesis of FFA from fructose, a single microreactor system for the formation of FFA from HMF was tested with a PTFE capillary tube $(500-\mu \mathrm{m}$ id, $1.6-\mathrm{mm}$ od, $100-\mathrm{cm}$ length, 60- $\mu$ l volume) using a magnetic-based Pd heterogeneous catalyst that was immobilized on the inside wall of the PTFE tube with several sets of external magnet rings (Figure 2).

The mesoporous SBA-15 silica $(10 \mathrm{~nm})$ deposited with Pd nanoparticles (3-5 nm) was initially synthesized and then further deposited with $\mathrm{Fe}_{3} \mathrm{O}_{4}$ particles $\left(30 \mathrm{~nm}\right.$ ) by adding $\mathrm{FeCl}_{2}$ and $\mathrm{FeCl}_{3}$ to form the mixed component type of $\mathrm{Pd} / \mathrm{Fe}_{3} \mathrm{O}_{4} / \mathrm{SiO}_{2}$ catalyst powder using the procedure reported earlier (Figure 2 and ESI, Supplementary Figure S4). ${ }^{5}$ Table 2 lists the results of the FFA synthesis via decarbonylation of commercial HMF under various reaction conditions. The conversion at $130{ }^{\circ} \mathrm{C}$ (entry $1-3$ ) increased from $73 \%$ (yield of $51 \%$ ) to $85 \%$ (yield of $61 \%$ ) as the residence time was varied from 5 to $12 \mathrm{~min}$ by manipulating the flow rate. Upon increasing the reaction temperature to $150{ }^{\circ} \mathrm{C}$ (entry 4-6), the conversion of FFA from HMF reached up to $99 \%$ with $95 \%$ yield for only $12 \mathrm{~min}$ of reaction time, which is comparable to $99 \%$ conversion with $95 \%$ selectivity obtained in the batch process under high pressure $(50 \mathrm{bar})$ at $180^{\circ} \mathrm{C}$ for $12 \mathrm{~h}$ in the presence of the Ir catalyst with phosphine ligand.

For the integration of the highly selective decarbonylation of HMF with the dehydration of D-fructose, a two-step, one-flow microreaction was then tested for directly synthesizing FFA from fructose by connecting the PTFE capillary to the end of the first dehydration of the silica-fused capillary, as illustrated in Figure 1. At a flow rate of $5 \mu \mathrm{l} \mathrm{min}{ }^{-1}$, the total residence time of $18 \mathrm{~min}(6 \mathrm{~min}$ in first reactor and $12 \mathrm{~min}$ in second reactor) at $150{ }^{\circ} \mathrm{C}$ resulted in a $99 \%$ conversion of fructose and $94 \%$ yield ( $88 \%$ of isolated yield) of FFA (entry 7 ). It is noteworthy that identical results were obtained with either commercial pure HMF or the as-produced HMF product without purification in the capillary microreactor. Moreover, to the best of our knowledge, this is the first report on the direct synthesis of FFA from carbohydrates. This remarkable performance highlights the integrated microfluidic process and system as a potential platform for biomass conversion. Note that rapid transport of the fragile HMF intermediate to the downstream caused no thermal loss even at high temperature. However, the activity of the $\mathrm{Pd} / \mathrm{Fe}_{3} \mathrm{O}_{4} / \mathrm{SiO}_{2}$ catalyst gradually degraded when the conversion of HMF to the FFA product was conducted at 


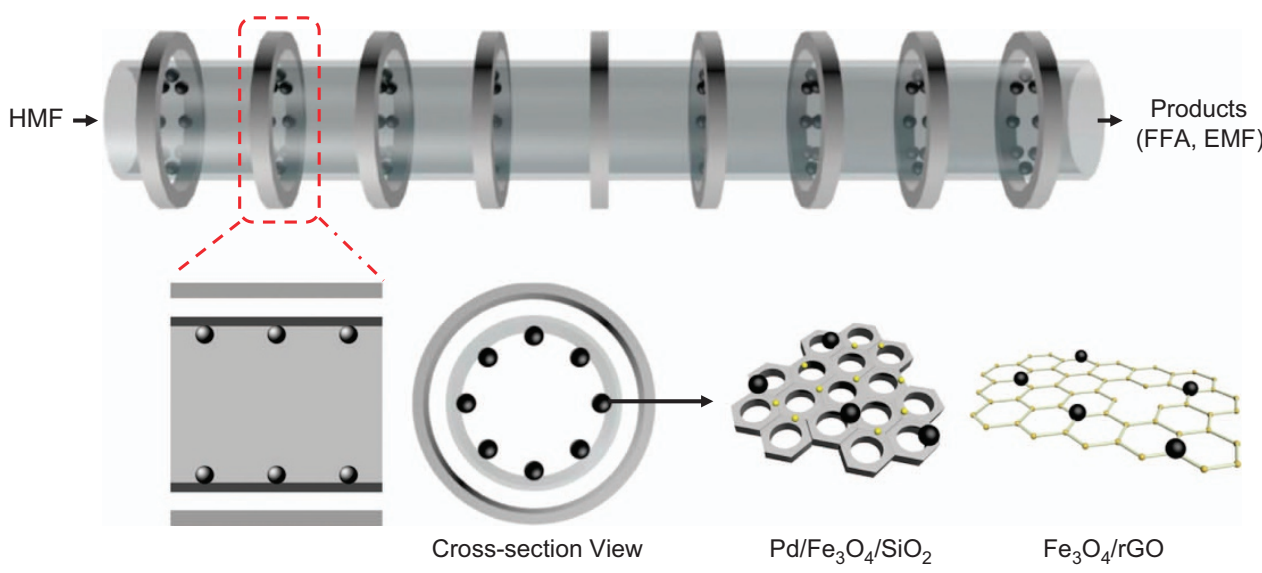

Figure 2 Illustrative scheme of binary-phase reactions for synthesis of FFA and EMF from HMF in different magnetic-based-catalyst-immobilized microfluidic systems. EMF, 5-ethoxymethylfurfural; FFA, furfuryl alcohol; HMF, 5-hydroxymethylfurfural.

Table 2 Catalytic decarbonylation of HMF into FFA in a PTFE capillary microreactor and directly from $\mathrm{D}$-fructose $(0.5 \mathrm{M}$ reagent in DMSO, $1 \mathrm{~g} \mathrm{Pd} / \mathrm{Fe}_{3} \mathrm{O}_{4} / \mathrm{SiO}_{2}$ catalysts)

\begin{tabular}{lcccc}
\hline Entry & Temp $\left({ }^{\circ} \mathrm{C}\right)$ & Time $(\mathrm{min})$ & Conv. (\%) & Yield (\%) \\
\hline 1 & 130 & 5 & 73 & 51 \\
2 & 130 & 9 & 82 & 55 \\
3 & 130 & 12 & 85 & 61 \\
4 & 150 & 5 & 95 & 75 \\
5 & 150 & 9 & 99 & 87 \\
6 & 150 & 12 & 99 & 95 \\
7 a & 150 & 12 & 99 & 94 \\
\hline
\end{tabular}

Abbreviations: Conv., conversion; DMSO, dimethylsulfoxide; FFA, furfuryl alcohol; HMF, 5hydroxymethylfurfural; Temp, temperature.

aWas performed directly from D-fructose in an integrated system, total 18-min residence time.

$150{ }^{\circ} \mathrm{C}$ for 2 days in the continuous-flow reaction (see ESI Supplementary Figure S5), although this result is comparable to previous results in the literature, and there was no leaching of $\mathrm{Pd}$ metal in the product mixture as measured by ICP-MS. ${ }^{5}$ Furthermore, to enhance the synthetic profile of the FFA, a scale-up synthesis was performed to obtain the gram order of the FFA product from HMF by increasing the flow rate in the extended capillary length with larger diameter (see ESI Supplementary Table S2). When synthesized for $10 \mathrm{~h}$ in an increased diameter to $1 \mathrm{~mm}$ from $500 \mu \mathrm{m}$, the isolated product by column chromatography was improved to $0.5 \mathrm{~g}$ at a flow rate $20 \mu \mathrm{min}^{-1}$ and $0.99 \mathrm{~g}$ at $40 \mu \mathrm{min}^{-1}$ ( $2 \mathrm{~m}$ of capillary). Finally, $1.49 \mathrm{~g}(18 \mathrm{mmol})$ of the FFA product with an isolated yield of $85 \%$ was obtained at $60 \mu \mathrm{min}^{-1}$ (3-m reactor, $720-\mu \mathrm{l}$ inner volume), demonstrating the scalable production efficiency by varying the experimental units with no complicated facility.

We then shifted our attention to the direct synthesis of EMF from fructose to diversify the usefulness and potential of the integrated microfluidic platform. From the 'green chemistry' concepts, the high energy density $\left(30.3 \mathrm{MJ}^{-1}\right)$ of EMF, similar to that of diesel $\left(33.6 \mathrm{MJ}^{-1}\right)$, has recently attracted much attention as an alternative vehicle fuel that could substitute for fossil fuels. ${ }^{24}$ In general, the synthesis of EMF by the etherification of HMF in the presence of ethanol has been conducted using various heterogeneous catalysts to render a broad range of yields (64-92\%) for several hours of reactions. ${ }^{25}$ Recently, an efficient one-pot approach for the formation of EMF from different carbohydrates was also reported with graphene oxide (GO) as a reusable and green catalyst. ${ }^{25}$

In this work, however, reduced GO (rGO) was used due to its better catalytic profile for various organic transformations against the GObased catalyst. ${ }^{26}$ Notably, the use of rGO as a catalyst has not been reported for an efficient etherification of HMF under mild conditions. For facile immobilization of the rGO by external magnet force, magnetic $\mathrm{Fe}_{3} \mathrm{O}_{4}$ nanoparticles were loaded on the rGO prepared by Hummer's method. ${ }^{26}$ After the immobilization of the $\mathrm{Fe}_{3} \mathrm{O}_{4} / \mathrm{rGO}$ mixture catalyst inside the wall of PTFE $(500-\mu \mathrm{m}$ id, $1.6-\mathrm{mm}$ od, 200$\mathrm{cm}$ length and 120- $\mu$ l volume), the commercial HMF and ethanol were introduced from the horizontal inlet and vertical inlet of the T-junction, respectively, into the PTFE capillary to optimize the conversion to EMF by varying the reaction conditions $\left(50-100^{\circ} \mathrm{C}\right.$, 6-18 min, see ESI, Supplementary Figure S1). At $100^{\circ} \mathrm{C}$, unstable microfluidic flow was observed, which was presumably due to the low boiling point of ethanol. When the reaction temperature was decreased to $70^{\circ} \mathrm{C}$, the HMF reacted with ethanol for etherification for a complete conversion to EMF with a $99 \%$ yield in only 6 min (flow rate of $5 \mu \mathrm{min}^{-1}$ ), which is superior to the $96 \%$ conversion and $92 \%$ yield of the conventional reaction at $100^{\circ} \mathrm{C}$ for $12 \mathrm{~h}$ with a GO catalyst. ${ }^{25}$ In particular, the batch process requires an autoclave type of reactor to avoid the evaporation of ethanol at $100^{\circ} \mathrm{C}$, whereas this capillary process allows an efficient reaction at $70^{\circ} \mathrm{C}$, presumably because of the fast heat transfer.

As a proof of concept, this etherification for the synthesis of EMF directly from fructose was performed using an integrated microreactor. The fructose/solvent mixture was injected at a flow rate of $5 \mu \mathrm{lmin}{ }^{-1}$ into the first silica capillary reactor at $150^{\circ} \mathrm{C}$ and was subsequently transferred to the second PTFE capillary reactor at $70^{\circ} \mathrm{C}$ by passing through a cooling bath to mix with the ethanol reagent introduced at a $5 \mu \mathrm{min}^{-1}$ flow rate (see ESI Supplementary Figure S1). The achievement of $99 \%$ conversion with a $99 \%$ yield of EMF ( $97 \%$ isolated yield) is an outstanding example of a clean chemical process with no side products and no waste. The durability of the rGO catalyst was confirmed by no significant change in the activity when the conversion of HMF to the EMF product was performed at $70{ }^{\circ} \mathrm{C}$ for 2 days in a continuous-flow manner (see ESI Supplementary Figure S5). The metal moiety in the reaction mixture was analyzed by ICP-MS to test for leaching during reactions for 2 days and was observed to be in the few p.p.m. range level in reactions. This direct biomass conversion with perfect selectivity via the HMF with a reactive 


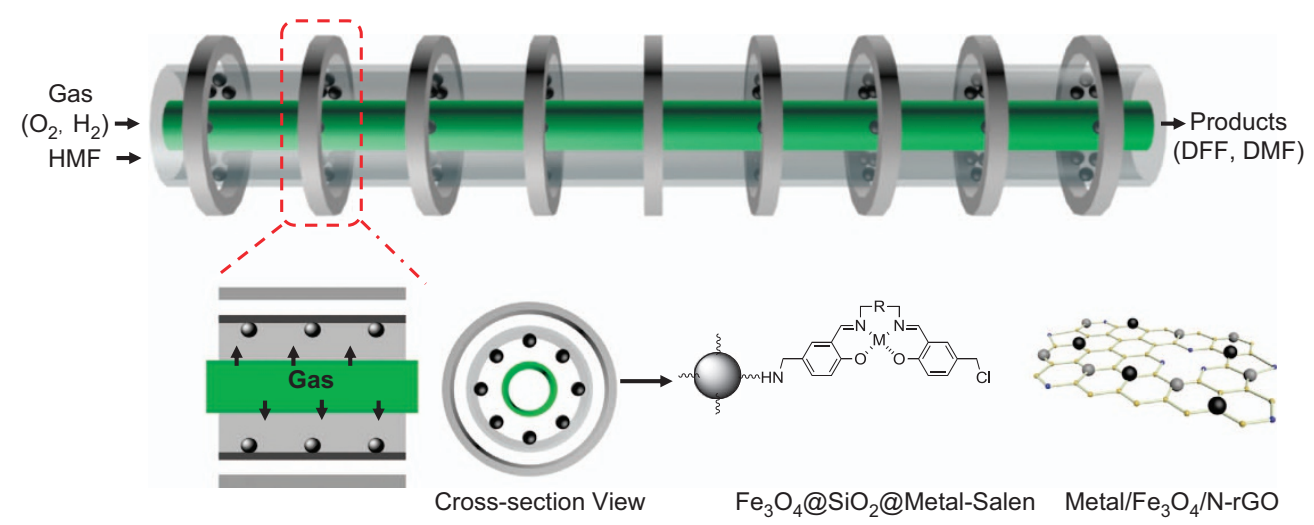

Figure 3 Illustrative scheme of gas-liquid-solid ternary phase reaction for oxidation and hydrogenolysis of HMF to DFF and DMF in a tube-in-tube microreactor. DFF, 2,5-diformylfuran; DMA, 2,5-dimethylfuran; HMF, 5-hydroxymethylfurfural.

Table 3 Selective oxidation of HMF to DFF in a tube-in-tube microreactor and directly from $\mathrm{D}$-fructose at $150^{\circ} \mathrm{C}(0.5 \mathrm{M}$ reagent in DMSO, 610- $\mu \mathrm{m}$ id, 750- $\mu \mathrm{m}$ od, 100-cm length of AF-2400, $46.8 \mathrm{~m} \mathrm{~min}^{-1}$ of $\mathrm{O}_{2}$ gas flow and $1 \mathrm{~g} \mathrm{Fe}_{3} \mathrm{O}_{4} @ \mathrm{SiO}_{2} @ \mathrm{Mn}$ salen-metal complex)

\begin{tabular}{lcccc}
\hline Entry & Types of salen ligand & Time (min) & Conv. (\%) & Yield (\%) \\
\hline 1 & 1 & 20 & $80(83)$ & $44(33)$ \\
2 & 1 & 40 & $81(85)$ & $46(37)$ \\
3 & 1 & 60 & $90(92)$ & $51(41)$ \\
4 & 2 & 20 & $88(91)$ & $75(46)$ \\
5 & 2 & 40 & $90(93)$ & $78(49)$ \\
6 & 2 & 60 & $93(95)$ & $84(52)$ \\
$7^{a}$ & 1 & $16 \mathrm{~h}$ & 69 & 34 \\
$8^{\mathrm{a}}$ & 2 & $16 \mathrm{~h}$ & 67 & 42 \\
$9^{\mathrm{b}}$ & 2 & $24 \mathrm{~h}$ & - & 77 \\
$10^{\mathrm{c}}$ & 2 & 70 & 92 & 82 \\
\hline
\end{tabular}

Abbreviations: Conv., conversion; DFF, 2,5-diformylfuran; DMSO, dimethylsulfoxide; HMF, 5-hydroxymethylfurfural.

Entries 1-10 were performed in the continuous-flow integrated microreactor for synthesizing DFF from commercial HMF.

Conversion and yield in parentheses (entries 1-6) with excessive flow of $\mathrm{O}_{2}$ gas

$(234.0 \mathrm{ml} \mathrm{min}-1$ of flow rate) in $810-\mu \mathrm{m}$ id, $1000-\mu \mathrm{m}$ od and $100-\mathrm{cm}$ length of AF-2400.

Type 1 and 2 salen ligand from ethylenediamine and trans-1,2-diaminocyclohexane, respectively.

In batch reactor

Reported data. ${ }^{30}$

COne-flow in an integrated system from D-fructose, total 70-min residence time.

furan ring and an aldehyde group was efficiently performed via precise control of the reaction temperature and time in each step in the integrated microfluidic system.

\section{Ternary phase conversion to furan chemicals from fructose}

The binary phase conversion of liquid reactants with a heterogeneous solid catalyst in a continuous-flow two-step microfluidic system has been established through the synthesis of FFA and EMF from fructose. Now, the integrated microfluidic approach can be further utilized for in situ chemical synthesis involving an HMF intermediate for the ternary phase conversion process of liquid-gas-solid. The selective oxidation of the hydroxyl group of HMF into the corresponding dialdehyde, DFF, has attracted much attention because of its versatility as a monomer of furan-based biopolymers and an intermediate of pharmaceuticals, antifungal agents and ligands. ${ }^{3}$ In general, the gasliquid reaction has been used for selective oxidation of HMF to DFF in the presence of a heterogeneous catalyst with molecular oxygen as an oxidant. ${ }^{6}$ For direct conversion to DFF from a carbohydrate in the conventional glass reactor, the two-step process requires careful isolation and purification of the HMF intermediate. ${ }^{3}$ Alternatively, the one-pot synthesis has suffered from insufficient mixing between the gas and liquid phase during the prolonged reaction, which often causes the formation of various side products. ${ }^{6}$ Therefore, strict control of the oxidation condition is strongly required for selective DFF production.

In this work, for the selective oxidation of HMF with oxygen gas and heterogeneous catalyst, a tube-in-tube microfluidic system as a gas/liquid/solid ternary phase microreactor was devised and fabricated, as illustrated in Figure 3 by positioning a gas-permeable inner Teflon tube $(610-\mu \mathrm{m}$ id, $750-\mu \mathrm{m}$ od, $100-\mathrm{cm}$ length of AF-2400) into the outer PTFE tube (1-mm id, $1.5-\mathrm{mm}$ od, $100-\mathrm{cm}$ length). The microporous (110-nm pore size) and chemical-resistant inner tube effectively delivered the oxygen gas as an oxidant to the HMF solution in the outer tube along the entire length. ${ }^{27-29}$ Magnetic salentransition metal core-shell catalysts (hereafter denoted $\mathrm{Fe}_{3} \mathrm{O}_{4} @$ $\left.\mathrm{SiO}_{2} @ \mathrm{~V}, \mathrm{Fe}_{3} \mathrm{O}_{4} @ \mathrm{SiO}_{2} @ \mathrm{Mn}\right)$ were prepared as reported elsewhere ${ }^{30}$ (see ESI Supplementary Scheme S1) and were immobilized on the inner wall of the PTFE tube with several sets of external magnet rings. Repeated use longer than $5 \mathrm{~h}$ in total did not cause release of the magnetic particles and no catalytic metal moiety in the product, indicating that the magnetic catalyst and metal were strongly bound on the inner wall and the particle surface, respectively.

Table 3 presents the results of the DFF synthesis via selective oxidation of HMF under various reaction conditions. The performance depended on the type of metal and ligand in the catalysts as well as the residence time. In the tube-in-tube system, the $\mathrm{Fe}_{3} \mathrm{O}_{4} @$ $\mathrm{SiO}_{2} @ \mathrm{Mn}$ complex catalyst from the 2-salen ligand exhibited the highest conversion of $93 \%$ with a $84 \%$ yield of DFF at $150{ }^{\circ} \mathrm{C}$ in $60 \mathrm{~min}$ of residence time, which is significantly superior to the performance of the $\mathrm{Fe}_{3} \mathrm{O}_{4} @ \mathrm{SiO}_{2} @ \mathrm{~V}$ catalyst (84\% conversion, 71\% yield) under the same reaction conditions (see ESI Supplementary Table S1). This excellent conversion in the tube-in-tube microreactor contrasts with the $67-69 \%$ conversion of the conventional batch reactions that required $\sim 16 \mathrm{~h}$ with purged oxygen through needles under identical conditions (entries 7-8 in Table 3 ) and the $77 \%$ yield obtained in $24 \mathrm{~h}$ of batch reaction with the identical $\mathrm{Fe}_{3} \mathrm{O}_{4} @ \mathrm{SiO}_{2} @ \mathrm{Mn}$ complex catalyst, as reported previously (entry 9). ${ }^{30}$ The stronger selective oxidation capability of the $\mathrm{Fe}_{3} \mathrm{O}_{4} @ \mathrm{SiO}_{2} @ \mathrm{Mn}$ catalyst from the 2-salen complex could presumably be ascribed to its favorable steric 
Table 4 Catalytic hydrogenolysis of HMF to DMF in a tube-in-tube microreactor and directly from D-fructose at $150{ }^{\circ} \mathrm{C}(0.5 \mathrm{M}$ reagent in DMSO, $610-\mu \mathrm{m}$ id, 750 -sm od, $100-\mathrm{cm}$ length of AF-2400, 8 bar $\mathrm{H}_{2}$ gas flow and $1 \mathrm{~g}$ metal/ $\mathrm{Fe}_{3} \mathrm{O}_{4} / \mathrm{N}$-rGO catalysts)

\begin{tabular}{|c|c|c|c|c|c|c|}
\hline \multirow[b]{2}{*}{ Entry } & \multirow[b]{2}{*}{ Catalyst } & \multirow[b]{2}{*}{ Conv. (\%) } & \multicolumn{4}{|c|}{ Yield } \\
\hline & & & $1 \mathrm{a}$ & $1 \mathrm{~b}$ & $1 \mathrm{c}$ & $\underset{1 d}{Y}$ \\
\hline 1 & $\mathrm{Ru}$ & 49 & 5 & - & 41 & 2 \\
\hline 2 & $\mathrm{Cu}$ & 81 & 10 & - & 50 & 8 \\
\hline 3 & Co & 71 & - & - & 12 & 54 \\
\hline 4 & $\mathrm{Pt}$ & 98 & 20 & 53 & - & - \\
\hline 5 & $\mathrm{Ru} / \mathrm{Co}_{0}$ & 81 & 49 & - & 13 & 12 \\
\hline 6 & $\mathrm{Cu} / \mathrm{Co}$ & 99 & 58 & - & 30 & - \\
\hline 7 & $\mathrm{Ru} / \mathrm{Pt}$ & 100 & 21 & 60 & 3 & 15 \\
\hline 8 & $\mathrm{Ru} / \mathrm{Cu}$ & 100 & 78 & 10 & - & - \\
\hline 9 & $\mathrm{Ru} / \mathrm{Cu}$ & 100 & 91 & - & - & - \\
\hline 10 & $\mathrm{Ru} / \mathrm{Cu}$ & 100 & 90 & - & - & - \\
\hline
\end{tabular}

Abbreviations: Conv., conversion; DMSO, dimethylsulfoxide; HMF, 5-hydroxymethylfurfural; N-rGO, N-doped graphene oxide.

Entries $1-8$ were performed in a high-pressure Parr reactor ( 30 bar $\mathrm{H}_{2}, 4 \mathrm{~h}$ ). Entry 9 for DMF from HMF and entry 10 for two-step one-flow DMF synthesis from D-fructose (26 min) in a tube-in-tube reactor (8 bar $\left.\mathrm{H}_{2}, 20 \mathrm{~min}\right)$

2,5-dimethylfuran (DMF, 1a), 2,5-dimethyltetrahydrofuran (1b), 2-methylfuran (1c) and 2,5-dihydroxymethylfuran (1d).

effect to oxidize the $-\mathrm{CH}_{2} \mathrm{OH}$ into $-\mathrm{CHO} .{ }^{30}$ The loaded amount of $\mathrm{Mn} / \mathrm{V}$ on the $\mathrm{Fe}_{3} \mathrm{O}_{4} @ \mathrm{SiO}_{2}$ was $50 \mathrm{mg}$ as quantified by TEM/EDX, the calculated TON (turnover number) value reached 157 for $\mathrm{Mn}$ and 360 for $\mathrm{V}$ at $150^{\circ} \mathrm{C}$ and $60 \mathrm{~min}$ of residence time, respectively. The durability of the $\mathrm{Fe}_{3} \mathrm{O}_{4} @ \mathrm{SiO}_{2} @ \mathrm{Mn}$ catalyst was confirmed by no significant change being observed in the activity during the initial $24 \mathrm{~h}$ when the conversion of HMF to the DFF product was performed at $150^{\circ} \mathrm{C}$ for 2 days in the tube-in-tube reactor (see ESI Supplementary Figure S5). In addition, we observed that an excessive amount of $\mathrm{O}_{2}$ flow through the inner tube led to a lower yield than an identical amount of $\mathrm{O}_{2}$ flow (entries 1-6 in parentheses). It is believed that the limited oxygen transport through the narrow diameter suppressed the formation of over-oxidation products in addition to the intimate contact of the gas-liquid-solid ternary phase in the tube-in-tube system, resulting in a higher selectivity of DFF.

Eventually, the continuous flow and direct conversion of carbohydrates to DFF was accomplished by integrating the two-step process of dehydration and oxidation in the assembled capillary microreactor, as shown in Figure 1. The diameters of the tubes in the two units were selected to accommodate the different reaction time needed in each reactor (see ESI Supplementary Figure S2). The total residence time of 70 min at $150{ }^{\circ} \mathrm{C}$ resulted in $92 \%$ conversion with $89 \%$ selectivity, indicating $82 \%$ yield to DFF based on the amount of fructose introduced in the system (entry 10). This overall conversion and selectivity delivered by the integrated system are nearly identical with the result $\left(93 \%\right.$ conversion, $90 \%$ selectivity, $60 \mathrm{~min}, 0.3 \mathrm{ml} \mathrm{h}^{-1}$ productivity) of the separate oxidative reaction. In addition, a negligible amount of furan-2,5-dicarboxylic acid as an overoxidation by-product was produced according to the GC-MS results, whereas the reaction at $150^{\circ} \mathrm{C}$ for $60 \mathrm{~min}$ appears to have converted $\sim 9 \%$ of HMF into an insoluble char product, as observed in the collection vial. In comparison, the best yield reported in the literature by a one-pot reaction with the stepwise addition of the two heterogeneous catalysts was $80 \%$ for $8 \mathrm{~h}$ at $110^{\circ} \mathrm{C}$ using a SBA$\mathrm{SO}_{3} \mathrm{H}$ and K-OMS-2 serial catalyst system, which required the separation of the catalyst from the product solution. ${ }^{8}$ However, the catalyst addition and separation steps were not needed in the integrated microfluidic process.
Encouraged by the ternary phase reaction in the tube-in-tube system, the challenging task of hydrogenolysis by $\mathrm{H}_{2}$ gas, which involves the hydrogenation of 2,5-functional groups of HMF to form dimethyl groups, followed by removing water molecules, was attempted. The synthesis of DMF from renewable sources has received considerable attention as an alternative liquid transportation fuel due to the energy density $\left(30 \mathrm{KJ} \mathrm{cm}^{-3}\right)$ with high octane number and ideal boiling point $\left(92^{\circ} \mathrm{C}\right) .^{7}$

In this work, N-rGO was selected as a catalyst support and metal aggregation preventer because the doped $\mathrm{N}$ atoms of $\mathrm{N}-\mathrm{rGO}$ provided preferable immobilization sites for the metal catalyst (see ESI Supplementary Figure S5). ${ }^{26}$ Table 4 presents the performance results of a series of mono- and bimetallic catalysts on a magnetic $\mathrm{Fe}_{3} \mathrm{O}_{4} / \mathrm{N}$ rGO support that were tested to evaluate the efficiency and selectivity of hydrogenolysis with hydrogen gas and heterogeneous catalyst in a tube-in-tube microfluidic system. A gas-permeable inner Teflon tube $(610-\mu \mathrm{m}$ id, $750-\mu \mathrm{m}$ od and $100-\mathrm{cm}$ length of AF-2400) was positioned into the outer PTFE tube $(1.2-\mathrm{mm}$ id, $1.5-\mathrm{mm}$ od and 100-cm length).

The metal-nanoparticle catalyst-decorated $\mathrm{Fe}_{3} \mathrm{O}_{4} / \mathrm{N}-\mathrm{rGO}$ was immobilized on the inner wall of the PTFE tube with several sets of external magnet rings (Figure 3). It is apparent that the mono- $\mathrm{Ru},-\mathrm{Cu}$ and -Co metal catalysts did not efficiently achieve the conversion of HMF into DMF at 30 bar $\mathrm{H}_{2}$ gas for $4 \mathrm{~h}$ in the high-pressure Parr reactor. The Pt metal catalyst nearly completed the conversion of HMF (98\%) with a low yield of DMF (20\%) due to the overhydrogenated product (1b, 53\% yield), as expected. ${ }^{31}$ In the bimetallic catalysts, the catalytic activity was generally higher than those of the monometallic catalysts. In particular, the $\mathrm{Ru} / \mathrm{Cu}$ catalyst exhibited the best activity with a $78 \%$ yield of DMF, assuring that the $\mathrm{Ru} / \mathrm{Cu} / \mathrm{Fe}_{3} \mathrm{O}_{4} /$ $\mathrm{N}$-rGO catalyst was suitable for the microfluidic hydrogenolysis reaction in the ternary phase. In entries 9 and 10 in Table 4, the continuous-flow microfluidic synthesis of DMF at 8 bar $\mathrm{H}_{2}$ gas led to a 91\% yield from commercial HMF for $20 \mathrm{~min}$ of residence time. The integrated one-flow process also showed a $90 \%$ yield from D-fructose for $26 \mathrm{~min}$ of total reaction time. The durability of the $\mathrm{Ru} / \mathrm{Cu} / \mathrm{Fe}_{3} \mathrm{O}_{4} /$ $\mathrm{N}-\mathrm{rGO}$ catalyst was confirmed by no significant change appearing in the activity when the conversion of HMF to DMF product was continuously performed at $150{ }^{\circ} \mathrm{C}$ for 2 days under 8 bar $\mathrm{H}_{2}$ gas in the 
tube-in-tube reactor (see ESI Supplementary Figure S5). In previous reports, homogeneous catalytic multi-step processes under harsh conditions (20-62 bar $\mathrm{H}_{2}$ ) resulted in much inferior results (45-78\% yields) and required catalyst recycle as well as a tedious separation-extraction step. ${ }^{32,33}$ Recently, the outstanding hydrogenolysis of HMF to DMF with a $98 \%$ yield at $180^{\circ} \mathrm{C}$ for $2 \mathrm{~h}\left(10 \mathrm{bar} \mathrm{H}_{2}\right.$ gas) was reported, in which bimetallic nanoparticles in hollow carbon nanospheres were used to avoid the aggregation of the nanometal catalyst. ${ }^{31}$ However, to the best our knowledge, our direct conversion from fructose to DMF is demonstrated for the first time with impressive performance under milder conditions, which is partly attributed to the efficient heat and mass transfer in the tube-in-tube microreactor. The overall conversion and yield of DMF directly from fructose by the integrated microfluidic transformation platform requires only simple operation and does not require any separation or removal steps of impurities from products. Moreover, the integrated microfluidic platform allowed safe use of highly pressurized explosive $\mathrm{H}_{2}$ gas.

\section{CONCLUSION}

In summary, an integrated continuous-flow microfluidic transformation platform was successfully demonstrated for the efficient and direct conversion of fructose biomass into heterocyclic furan derivatives via in situ utilization of HMF by a sequential two-step heterogeneous catalytic process. This tandem strategy integrated the complete dehydration of fructose into HMF by a surface sulfonic acid catalyst at $150^{\circ} \mathrm{C}$ for $6 \mathrm{~min}$ with subsequent binary phase reactions of decarbonylation and etherification or ternary liquid-gas-solid phase reactions of oxidation and hydrogenolysis of HMF into respective furan compounds. The remarkable $94 \%$ yield of FFA and $99 \%$ yield of EMF were achieved via the decarbonylation and etherification of HMF by $\mathrm{Pd} / \mathrm{Fe}_{3} \mathrm{O}_{4} / \mathrm{SiO}_{2}$ at $150^{\circ} \mathrm{C}$ for $12 \mathrm{~min}$ and the $\mathrm{Fe}_{3} \mathrm{O}_{4} / \mathrm{rGO}$ catalyst at $70^{\circ} \mathrm{C}$ for $6 \mathrm{~min}$, respectively. Furthermore, this transformation platform was extended to ternary oxidation and hydrogenolysis reactions in the tube-in-tube system for the production of DFF in $82 \%$ yield and DMF in 90\% yield by $\mathrm{Fe}_{3} \mathrm{O}_{4} @ \mathrm{SiO}_{2} @ \mathrm{Mn}$ for $70 \mathrm{~min}$ and $\mathrm{Ru} / \mathrm{Cu} / \mathrm{Fe}_{3} \mathrm{O}_{4} /$ $\mathrm{N}$-rGO catalyst for $26 \mathrm{~min}$ at $150^{\circ} \mathrm{C}$, respectively. In particular, the supported catalysts on the magnetic $\mathrm{Fe}_{3} \mathrm{O}_{4}$ particles enabled easy positioning of an appropriate amount of catalyst at the inner wall of the microchannel using an external magnet. This innovative one-flow transformation platform led to superior performance of the direct biomass conversion compared with batch reactions because of the precise control of the reaction temperatures and times in a time-,costand labor-saving manner with no catalyst recycle and no tedious separation-extraction step. It is envisaged that this concept and these approaches can be further expanded to multicomponent or cascade transformation of natural lignocellulosic biomass resources to meet industrial requirements and to ultimately help solve the energy problem in the future.

\section{CONFLICT OF INTEREST}

The authors declare no conflict of interest.

\section{ACKNOWLEDGEMENTS}

This work was supported by a National Research Foundation of Korea (NRF) grant funded by the Korean Government (MEST) (2008-0061983).
1 Kunkes, E. L., Simonetti, D. A., West, R. M., Serrano-Ruiz, J. C., Gartner, C. A. \& Dumesic, J. A. Catalytic conversion of biomass to monofunctional hydrocarbons and targeted liquid-fuel classes. Science 322, 417-421 (2008).

2 Xie, H. B., Zhao, Z. K. \& Wang, Q. Catalytic conversion of inulin and fructose into 5 hydroxymethylfurfural by lignosulfonic acid in ionic liquids. ChemSusChem $\mathbf{5}$, 901-905 (2012).

3 Van Putten, R. J., Van der Waal, J. C., Jong, E., Rasrendra, C. B., Heeres, H. J. \& Vries, J. G. Hydroxymethylfurfural, a versatile platform chemical made from renewable resources. Chem. Rev. 113, 1499-1597 (2013).

4 Roman-Leshkov, Y., Chheda, J. N. \& Dumesic, J. A. Phase modifiers promote efficient production of hydroxymethylfurfural from fructose. Science 312, 1933-1937 (2006).

5 Huang, Y. B., Yang, Z., Chen, M.-Y., Dai, J.-J., Guo, Q.-X. \& Fu, Y. Heterogeneous palladium catalysts for decarbonylation of biomass-derived molecules under mild conditions. ChemSusChem 6, 1348-1351 (2013).

6 Halliday, G. A., Young, R. J. \& Grushin, V. V. One-pot, two-step, practical catalytic synthesis of 2,5-diformylfuran from fructose. Org. Lett. 5, 2003-2005 (2003).

7 Roman-Leshkov, Y., Barrett, C. J., Liu, Z. Y. \& Dumesic, J. A. Production of dimethylfuran for liquid fuels from biomass-derived carbohydrates. Nature 447, 982-U985 (2007)

8 Yang, Z. Z., Deng, J., Pan, T., Guo, Q. X. \& Fu, Y. A one-pot approach for conversion of fructose to 2,5-diformylfuran by combination of Fe304-SBA-SO3H and K-OMS-2. Green Chem. 14, 2986-2989 (2012).

9 Takagaki, A., Takahashi, M., Nishimura, S. \& Ebitani, K. One-pot synthesis of 2,5-diformylfuran from carbohydrate derivatives by sulfonated resin and hydrotalcitesupported ruthenium catalysts. Acs Catal. 1, 1562-1565 (2011).

10 Broadwater, S. J., Roth, S. L., Price, K. E., Kobaslija, M. \& McQuade, D. T. One-pot multi-step synthesis: a challenge spawning innovation. Org. Biomol. Chem. 3, 2899-2906 (2005).

11 Toure, B. B. \& Hall, D. G. Natural product synthesis using multicomponent reaction strategies. Chem. Rev. 109, 4439-4486 (2009).

12 Maurya, R. A., Park, C. P., Lee, J. H. \& Kim, D. P. Continuous in situ generation, separation, and reaction of diazomethane in a dual-channel microreactor. Angew. Chem. Int. Ed. 50, 5952-5955 (2011).

13 Basavaraju, K. C., Sharma, S., Maurya, R. A. \& Kim, D. P. Safe use of a toxic compound: heterogeneous $\mathrm{OsO} 4$ catalysis in a nanobrush polymer microreactor. Angew. Chem. Int. Ed. 52, 6735-6738 (2013).

14 Sharma, S., Maurya, R. A., Min, K. I., Jeong, G. Y. \& Kim, D. P. Odorless isocyanide chemistry: an integrated microfluidic system for a multistep reaction sequence. Angew. Chem. Int. Ed. 52, 7564-7568 (2013).

15 Webb, D. \& Jamison, T. F. Continuous flow multi-step organic synthesis. Chem. Sci. 1, 675-680 (2010).

16 Whitesides, G. M. The origins and the future of microfluidics. Nature 442 , 368-373 (2006)

17 Thorsen, T., Maerkl, S. J. \& Quake, S. R. Microfluidic large-scale integration. Science 298, 580-584 (2002).

18 Jahnisch, K., Hessel, V., Lowe, H. \& Baerns, M. Chemistry in microstructured reactors. Angew. Chem. Int. Ed. 43, 406-446 (2004).

$19 \mathrm{Kim}, \mathrm{H}$., Nagaki, A. \& Yoshida, J. A flow-microreactor approach to protecting-group-free synthesis using organolithium compounds. Nat. Commun. 2, 264 (2011).

20 Marre, S. \& Jensen, K. F. Synthesis of micro and nanostructures in microfluidic systems. Chem. Soc. Rev. 39, 1183-1202 (2010).

21 Gill, C. S., Price, B. A. \& Jones, C. W. Sulfonic acid-functionalized silica-coated magnetic nanoparticle catalysts. J. Catal. 251, 145-152 (2007).

22 Bae, J. A., Song, K.-C., Jeon, J.-K., Ko, Y. S., Park, Y.-K. \& Yim, J.-H. Effect of pore structure of amine-functionalized mesoporous silica-supported rhodium catalysts on 1octene hydroformylation. Micropor. Mesopor. Mat. 123, 289-297 (2009).

23 Geilen, F. M. A., Stein, T. V., Engendahl, B., Winterle, S., Liauw, M. A., Klankermayer, J. \& Leitner, W. Highly selective decarbonylation of 5-(hydroxymethyl) furfural in the presence of compressed carbon dioxide. Angew. Chem. Int. Ed. 50, 6831-6834 (2011).

24 Liu, A. Q., Liu, B., Wang, Y. M., Ren, R. S. \& Zhang, Z. H. Efficient one-pot synthesis of 5-ethoxymethylfurfural from fructose catalyzed by heteropolyacid supported on K-10 clay. Fuel 117, 68-73 (2014).

25 Wang, H. L., Deng, T., Wang, Y., Cui, X., Qi, Y., Mu, X., Hou, X. \& Zhu, Y. Graphene oxide as a facile acid catalyst for the one-pot conversion of carbohydrates into 5-ethoxymethylfurfural. Green Chem. 15, 2379-2383 (2013).

26 Su, C. L., Acik, M., Takai, K., Lu, J., Hao, S.-J., Zheng, Y., Wu, P., Bao, Q., Enoki, T., Chabal, Y. J. \& Loh, K.P. Probing the catalytic activity of porous graphene oxide and the origin of this behaviour. Nat. Commun. 3, 1298 (2012).

27 Petersen, T. P., Polyzos, A., O'Brien, M., Ulven, T., Baxendale, I. R. \& Ley, S. V. The oxygen-mediated synthesis of 1,3-butadiynes in continuous flow: using Teflon AF-2400 to effect gas/liquid contact. ChemSusChem 5, 274-277 (2012).

28 Polyzos, A., O'Brien, M., Petersen, T. P., Baxendale, I. R. \& Ley, S. V. The continuousflow synthesis of carboxylic acids using $\mathrm{CO} 2$ in a tube-in-tube gas permeable membrane reactor. Angew. Chem. Int. Ed. 50, 1190-1193 (2011).

29 O'Brien, M., Taylor, N., Polyzos, A., Baxendale, I. R. \& Ley, S. V. Hydrogenation in flow: Homogeneous and heterogeneous catalysis using Teflon AF-2400 to effect gas-liquid contact at elevated pressure. Chem. Sci. 2, 1250-1257 (2011).

30 Amarasekara, A. S., Green, D. \& McMillan, E. Efficient oxidation of 5-hydroxymethylfurfural to 2,5-diformylfuran using $\mathrm{Mn}(\mathrm{III})$-salen catalysts. Catal. Commun. 9, 286-288 (2008). 
31 Wang, G. H., Hilgert, J., Richter, F. H., Wang, F., Bongard, H.-J., Spliethoff, B., Weidenthaler, C. \& Schuth, F. Platinum-cobalt bimetallic nanoparticles in hollow carbon nanospheres for hydrogenolysis of 5-hydroxymethylfurfural. Nat. Mater. 13 294-301 (2014).

32 Thananatthanachon, T. \& Rauchfuss, T. B. Efficient production of the liquid fuel 2,5-dimethylfuran from fructose using formic acid as a reagent. Angew. Chem. Int. Ed. 49, 6616-6618 (2010).

33 Binder, J. B. \& Raines, R. T. Simple chemical transformation of lignocellulosic biomass into furans for fuels and chemicals. J. Am. Chem. Soc. 131, 1979-1985 (2009). (c) (i) This work is licensed under a Creative Commons Attribution 4.0 International License. The images or other third party material in this article are included in the article's Creative Commons license, unless indicated otherwise in the credit line; if the material is not included under the Creative Commons license, users will need to obtain permission from the license holder to reproduce the material. To view a copy of this license, visit http:// creativecommons.org/licenses/by/4.0/

Supplementary Information accompanies the paper on the NPG Asia Materials website (http://www.nature.com/am) 